\title{
Synthesis and Electric Conductivity Study of 4-(p-Aminophenylazo) Benzene Sulphonic Acid
}

\author{
Ramadan Ali Bawa, Ebtissam Jommah Zaghloot, Khadijah Etohammi Mossa and Najjat Omran Issa \\ Department of Chemistry, Faculty of Science, Misurata University, Misurata, Libya
}

Received: October 23, 2012 / Accepted: November 16, 2012 / Published: March 25, 2013.

\begin{abstract}
An azo dye derived from sulphanilic acid was synthesized in moderate yield by a coupling reaction between a diazonium salt of the sulphanilic acid and aniline at $0-5{ }^{\circ} \mathrm{C}$. The resulting azo derivative was characterized using nuclear magnetic resonance (NMR) and various spectroscopic methods such as uv-vis, infrared radiation (IR) and ms. The electric conductivity $\sigma$ of the resulting 4- $\left(p\right.$-aminophenylazo) benzene sulphonic acid was determined and found to be $0.917875 \times 10^{-7}(\Omega \cdot \mathrm{cm})^{-1}$. The 4 - $(p$-aminophenylazo) benzene sulphonic acid showed a semiconducting behavior at $295 \mathrm{~K}$ without the need of adding a doping agent. The electric conductivity measurements revealed that the synthesized 4-( $p$-aminophenylazo) benzene sulphonic acid possesses an electric resistivity $\rho$ of $1.089472779 \times 10^{7} \Omega \cdot \mathrm{cm}$ which renders this azo dye a semiconducting material.
\end{abstract}

Key words: Azo dye, synthesized, electric conductivity, semiconducting.

\section{Introduction}

Azo dyes have been of great interest of many research groups due to their stability, ease of synthesis and electrical properties [1, 2]. They provide a wide range of colours that depend on the number of azo groups and the substituents on the aryl moieties. These colors are originated from absorbing light in the visible region of the spectrum, making use of the delocalization of $n$ - and $\pi$-electrons throughout their structures [1, 3]. The main difference between semiconductors and good conductors is that the resistance of the latter declines rapidly as the temperature decreases, whereas the resistance of semiconductors increases remarkably as the temperature falls down to the absolute zero [4]. The structure of the semiconductors allows electrical current to pass through and they possess electrical resistivity ranging between $10^{-2} \Omega \cdot \mathrm{cm}$ and $10^{9} \Omega \cdot \mathrm{cm}$ at room temperature [4-7]. The d.c. electric conductivity of azo dyes has been investigated [8]. The factors that

Corresponding author: Ramadan Ali Bawa, Ph.D., assistant professor, research fields: organic synthesis and organometallics. E-mail: ramadanali70@yahoo.com. are affecting the electric conductivity have been intensively studied by a number of research groups [1, 2 , 9]. Here in, an azo dye, 4-( $p$-aminophenylazo) benzene sulphonic acid, has been synthesized and its electric conductivity at $295 \mathrm{~K}$ has also been determined.

\section{Materials and Methods}

\subsection{Materials}

Sulphanic acid, sulphuric acid and sodium nitrite were purchased from Riedel-Dehean. The aniline was obtained from Hassco Belgium. These chemicals were used without further purification.

\subsection{Instrumentation}

Melting point was measured on a UK Bamstead/Electro thermal-9200 apparatus and is uncorrected. Uv-vis absorptions were recorded on Uv-vis spectrophotometer-uv mini 1240-Shimadzu. pH was measured using Jenway $\mathrm{pH}$ meter $3505 .{ }^{1} \mathrm{H}$ NMR spectrum was recorded on a Bruker Avance 300 spectrometer. Residual proton signals from the deuteriated solvents were used as references [DMSO 
$\left.\left({ }^{1} \mathrm{H}, 2.50 \mathrm{ppm}\right)\right]$. Coupling constants were measured in Hz. Infrared spectrum was recorded on Jasco FT/IR-4100 Fourier transform infrared spectrometer. Mass spectrum was recorded on a Micromass Autospec M spectrometer. The electric measurements were carried out using Laboratory power supply EA-PS 2016-050. The electrical current was measured in $\mu \mathrm{A}$ units by the use of CEM/DT-3900 ammeter. The applied voltage was determined in $\mathrm{V}$ units by employing Peak Tech 2010 DMM voltmeter.

\subsection{Preparation of 4-(p-aminophenylazo) benzene sulphonic acid 3}

An adapted literature procedure [10], was followed towards the synthesis of the azo derivative 3. A solution of sulphanilic acid $(3.72 \mathrm{~g}, 20 \mathrm{mmol})$ in $2 \mathrm{~N}$ sulphuric acid $\left(272 \mathrm{~cm}^{3}\right)$ was cooled down to $0-5{ }^{\circ} \mathrm{C}$. An aqueous solution of sodium nitrite $(5.44 \mathrm{~g}, 2.54$ mmol in $22 \mathrm{~cm}^{3}$ water) was added slowly to the cooled sulphanilic acid solution maintaining the temperature at $0-5{ }^{\circ} \mathrm{C}$ while stirring. Freshly purified aniline $(2.00 \mathrm{~g}$, $20 \mathrm{mmol}$ ) was then added to the reaction mixture with a continuous stirring at $0-5{ }^{\circ} \mathrm{C}$ for $30 \mathrm{~min}$. The reaction mixture was left standing overnight at room temperature after which the reaction mixture was treated by a solution of ammonium hydroxide $\left(8 \mathrm{~cm}^{3}, 2 \mathrm{~N}\right)$ raising the $\mathrm{pH}$ up to about 6 . A reddish brown precipitate of the desired azo dye was formed, filtered and air-dried to give $62 \%$ yield of the azo derivative 3 (3.69 g, $21.52 \mathrm{mmol}$ ). No further purification was required. mp $290{ }^{\circ} \mathrm{C}$ (dec); UV (n-propyl alcohol) $434.0 \mathrm{~nm}$; IR (KBr) vcm ${ }^{-1}, 3,336\left(\mathrm{NH}_{2}\right), 1,417(\mathrm{~N}=\mathrm{N})$, $1,020(\mathrm{~S}=\mathrm{O}) .{ }^{1} \mathrm{H}$ HNMR $\delta_{\mathrm{H}}\left[300 \mathrm{MHz} ;\left(\mathrm{CD}_{3}\right)_{2} \mathrm{SO}\right]$ 7.82-7.76 (6H, m, ArH); 6.96-6.93 (2H, m, ArH); 3.40 (3H, br s, $\mathrm{NH}_{2}$ and $\left.\mathrm{SO}_{3} \mathrm{H}\right)$. MS $m / z \mathrm{EI}^{+}(\mathrm{M}+1$, $\mathrm{C}_{12} \mathrm{H}_{12} \mathrm{~N}_{3} \mathrm{O}_{3} \mathrm{~S}$, 278.06) 279 (17), 278 (35), 144 (260), 143 (22), 93 (100), 81 (35), 80 (83), 64 (70).

\subsection{General Procedure for Measuring the Electric} Current

An adapted literature procedure was followed [11, 12].
The sample of the azo dye 3 (with a radius of $0.25 \mathrm{~cm}$ and thickness of $0.1 \mathrm{~cm}$ ) was placed between two copper electrodes one of which was connected to a power source and the other copper electrode was connected to an ammeter to measure the electrical current. The electric measurements were carried out at $295 \mathrm{~K}$.

\section{Results and Discussion}

The 4-( $p$-aminophenylazo) benzene sulphonic acid 3 was synthesized from a coupling reaction between the diazonium bisulphate salt of the sulphanilic acid 1 and the aniline 2 at $0-5{ }^{\circ} \mathrm{C}$ (Scheme 1). The wavelength for the uv-vis absorption of the resulting azo dye 3 was measured and found to be $434.0 \mathrm{~nm}$ in $n$-propyl alcohol.

The IR spectrum also showed the vibrational band of the azo group at $1,417 \mathrm{~cm}^{-1}[10,13]$.

A packed pellet of the azo dye 3, with a radius of $0.25 \mathrm{~cm}$ and a thickness of $0.1 \mathrm{~cm}$, was placed between two copper electrodes and subjected to a voltage ranging between 0.1-4.0 V. The passed electrical current through the azo dye pellet, at $295 \mathrm{~K}$, was measured in Table 1.

These experimental results were processed by ORIGIN 8.1 and it was found that the correlation between the applied voltage and the measured current is linear relationship (Fig. 1) following the Eq. (1).

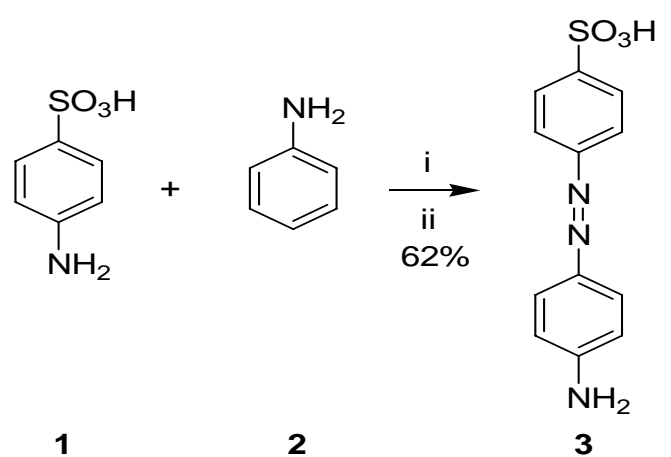

Reagents and conditions: (i) aq. $\mathrm{NaNO}_{2}$, conc. $\mathrm{H}_{2} \mathrm{SO}_{4}, \mathrm{O}-5^{\circ} \mathrm{C}, 1 \mathrm{~h}$; (ii) stirring, $\mathrm{O}-5^{\circ} \mathrm{C}, 30$ min, then rt, $24 \mathrm{~h}$

Scheme 1 Synthesis of 4-(p-aminophenylazo) benzene sulphonic acid 3. 
Table $1 \mathrm{~V}=$ the applied voltage value in volt unit; $A=$ the measured current in ampere unit.

\begin{tabular}{llllll}
\hline $\mathrm{V}$ & $\begin{array}{l}\mathrm{A} \\
\times 10^{-7}\end{array}$ & $\mathrm{~V}$ & $\begin{array}{l}\mathrm{A} \\
\times 10^{-7}\end{array}$ & $\mathrm{~V}$ & $\begin{array}{l}\mathrm{A} \\
\times 10^{-7}\end{array}$ \\
\hline 0.1 & 0.1 & 1.3 & 2.3 & 2.9 & 5.1 \\
0.2 & 0.2 & 1.4 & 2.5 & 3.0 & 5.3 \\
0.3 & 0.4 & 1.5 & 2.7 & 3.1 & 5.5 \\
0.4 & 0.6 & 1.6 & 2.8 & 3.2 & 5.7 \\
0.5 & 0.8 & 1.7 & 2.9 & 3.3 & 5.9 \\
0.6 & 0.9 & 1.8 & 3.1 & 3.4 & 6.1 \\
0.7 & 1.1 & 1.9 & 3.3 & 3.5 & 6.3 \\
0.8 & 1.3 & 2.0 & 3.5 & 3.6 & 6.5 \\
0.9 & 1.5 & 2.1 & 3.8 & 3.7 & 6.6 \\
1.0 & 1.7 & 2.2 & 3.9 & 3.8 & 6.7 \\
1.1 & 1.9 & 2.3 & 4.1 & 3.9 & 6.8 \\
1.2 & 2.1 & 2.4 & 4.2 & 4.0 & 6.9 \\
\hline
\end{tabular}

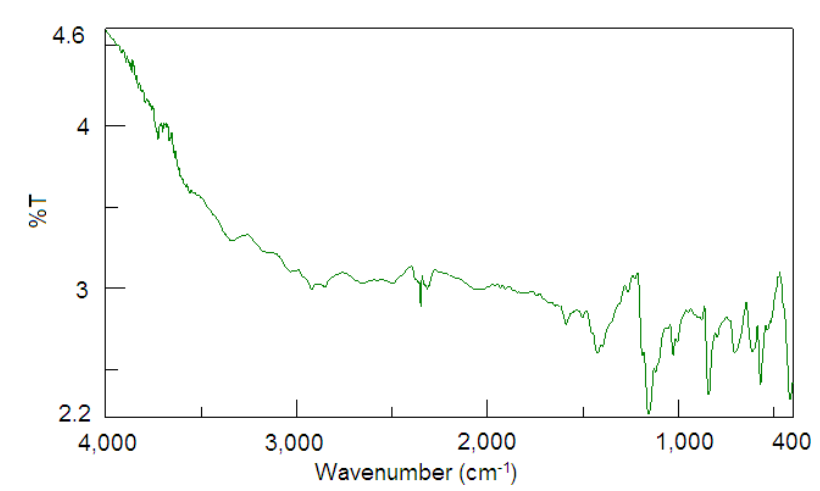

Fig. 1 The IR spectrum of the azo dye 3.

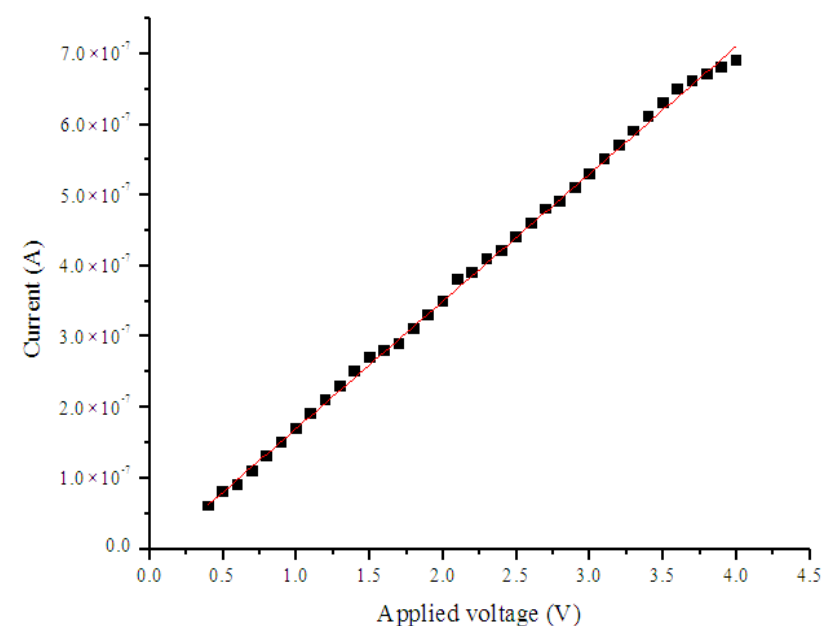

Fig. 2 The applied voltage (V) vs. current (A).

$$
y=a+b x
$$

The resistance $R$ equals the reverse of the slope $\left(\right.$ slope $\left.=1.80133 \times 10^{-7} \Omega^{-1}\right)($ Eq. (2)):

$$
\begin{gathered}
R=\frac{1}{1.80133 \times 10^{-7} \Omega^{-1}} \\
\therefore R=5.55145365 \times 10^{6}(\Omega)
\end{gathered}
$$

The electric resistivity $\rho$ of the azo dye 3 could easily be determined Eq. (3).

$$
\rho=R \frac{A}{l}
$$

where $A$ is the surface area of the sample pellet and equals $\pi r^{2}$ ( $r=0.25 \mathrm{~cm}$ ), whereas $l$ is the thickness of the sample pellet and equals $0.1 \mathrm{~cm}$ :

$$
\begin{gathered}
\frac{A}{l}=\frac{\pi r^{2}}{l} \\
\therefore \frac{A}{l}=\frac{3.14 \times(0.25 \mathrm{~cm})^{2}}{0.1 \mathrm{~cm}}=1.9625 \mathrm{~cm} \\
\therefore \rho=5.55145365 \times 10^{6} \Omega \times 1.9625 \mathrm{~cm} \\
\rho=1.089472779 \times 10^{7} \Omega \cdot \mathrm{cm}
\end{gathered}
$$

The electric resistivity $\rho$ (Eq. (5)) of the azo dye 3 comes within the range of the semiconductors' electric resistivity which is between $10^{-2} \Omega \cdot \mathrm{cm}$ and $10^{9} \Omega \cdot \mathrm{cm}$ [4]. Therefore, the electric conductivity $\sigma$ of the azo dye 3 could easily be calculated :.

$$
\sigma=\frac{1}{\rho}=0.917875 \times 10^{-7} \Omega^{-1} \mathrm{~cm}^{-1}
$$

\section{Conclusions}

The azo dye, 4-(p-aminophenylazo) benzene sulphonic acid 3, was synthesized in rather good yield. This azo dye was characterized using various spectroscopic techniques. The electric conductivity study on this azo dye revealed that the 4-( $p$-aminophenylazo) benzene sulphonic acid 3 could be classified as a semiconductor with an electric resistivity $\rho$ of $1.089472779 \times 10^{7} \Omega \cdot \mathrm{cm}$ which is within the range of the semiconducting materials.

\section{Acknowledgments}

The authors would like to thank the Department of Chemistry and the Research Centre at the Misurata University for facilitating the essential needs that were required for this project. The authors' thanks also go to Esra M. Benhmaida, Hanna M. Asha-Affi and Hyffa A. Alamin from the Department of Physics for their kind assistance.

\section{References}

[1] M. Aziz, H. El-Mallah, Electrical and optical properties of azo dye, Indian Journal of Pure and Applied Physics 47 (2009) 530-534. 
[2] M. Aziz, A. El-Sonbati, A. Hilali, D.C. Conduction phenomenon of some rhodanine azo complexes, Chem. Pap. 56 (2002) 305-308.

[3] S. Yin, H. Xu, W. Shi, Y. Gao, Y. Song, J. Lam, et al., Synthesis and optical properties of polyacetylenes containing nonlinear optical chromophores, Polymer 46 (2005) 7670-7677.

[4] C. Kittel, Introduction to Solid State Physics 5th ed, Jonhn Wiley \& Sons, New York, USA, 1976, p. 207.

[5] R. Bawa, Electric conductivity study of o-substituted phenoxo iron (III) complexes, Journal of Chemistry and Chemical Engineering 4 (2010) 54-58.

[6] R. Bawa, Preparation and study of some arylamino iron (III) complexes, Journal of Materials Science and Engineering 3 (2009) 51-53.

[7] R. Bawa, Structure determination and electric conductivity of phenolic iron (III) complexes, Alsatil Journal 3 (2009) 61- 69.

[8] M. Abd El Wahed, S. Aly, H. Farouk, Electrical properties of some binuclear azo complexes, Journal of Materials Science Letters 13 (1994) 1434-1436.

[9] R. Salih, Synthesis, identification and study of electrical conductivity of the doped poly $p$-anisidine, J. Mater. Environ. Sci. 3 (2012) 50-56.

[10] I. Mohammed, A. Mustapha, Synthesis of new azo compounds based on $N$-(4-hydroxypheneyl)maleimide and $N$-(4-methylpheneyl) maleimide, Molecules 15 (2010) 7498-7508.

[11] D. Halliday, R. Resnick, J. Walker, Fundamentals of Physics, 7th ed., John Wiley \& Sons Inc, Von Hoffmann Press, Hoboken, NJ, USA, 2005, pp. 693-697.

[12] J. Beddah, J. Alfurdq, Applications of Practical Physics, 1st ed., Dar Alhekmah \& Dar Assaria Publishers, Valletta, Malta, 2003, pp. 95-98.

[13] S. Hameed, Characterization and thermal decomposition of indolylidine aniline azo dyes derivatives, Jordan J. Chem. 2 (2007) 133-144. 